ÉGYPTE

monde arabe

\section{Égypte/Monde arabe}

7| 1991

Perceptions de la centralité de l'Égypte 1

\title{
Un tour en ville
}

\section{Youssef Idris}

Traducteur : Philippe Vigreux

\section{CpenEdition Journals}

Édition électronique

URL : https://journals.openedition.org/ema/1195

DOI : 10.4000/ema. 1195

ISSN : 2090-7273

Éditeur

CEDEJ - Centre d'études et de documentation économiques juridiques et sociales

Édition imprimée

Date de publication : 30 septembre 1991

Pagination : 191-201

ISSN : 1110-5097

Référence électronique

Youssef Idris, «Un tour en ville », Égypte/Monde arabe [En ligne], 7 | 1991, mis en ligne le 08 juillet 2008, consulté le 07 juillet 2022. URL : http://journals.openedition.org/ema/1195 ; DOI : https://doi.org/ 10.4000/ema. 1195

Ce document a été généré automatiquement le 7 juillet 2022

Tous droits réservés 


\section{Un tour en ville}

\section{Youssef Idris}

Traduction : Philippe Vigreux

\section{NOTE DE L'ÉDITEUR}

Traduction de l'arabe et notes par Philippe Vigreux. Cette nouvelle, tirée du recueil 'Arkhas layâlî, a été publiée pour la première fois au Caire en 1954, sous le titre « Meshwâr ».

1 À peine évoquait-on Le Caire, au fil d'une discussion, Chabrâwi restait transi et voyait bien que sa vie n'était pas une vie. Il languissait d'une heure passée à al-'Obêsî ou chez maître Ahmed, à al-Turgumân ${ }^{1}$, ruminant sa nostalgie des matinées du cinéma al-Ahlî² Son esprit s'envolait vers les jours d'autrefois, du temps de l'armée, où il arpentait Le Caire chaque semaine, de long en large.

2 La plupart du temps, il couronnait ses soupirs, ses regrets et ses désirs d'un vœu qu'il n'eût guère coûté à Dieu d'exaucer : lui fournir l'occasion, deux ou trois sous, et puis... cingler vers la ville pour revivre un de ces jours d'autrefois. Les collègues l'identifiaient à sa phrase, tant de fois répétée : «O Caire, je donnerais ma vie pour une heure dans tes bras!»

3 Mais il n'eut pas besoin de donner sa vie. La bénédiction tomba hors de toute attente et par une voie qu'il n'avait en rien soupçonnée.

4 Un jour qu'il était assis au poste, à sa manière habituelle depuis quatre ans, arriva soudain toute une compagnie. Une question posée, un brouhaha, et il apparut qu'il s'agissait d'une femme demeurée, originaire de Kafr Gom'a, accompagnée de ses proches, de sa belle-famille et des voisins. Le tumulte emplit l'endroit, on se pressa de plus belle et le poste devint trop petit.

5 Le coeur de Chabrâwi se mit à palpiter d'espoir.

6 Inévitablement, il allait falloir envoyer la femme au Caire, à l'hôpital psychiatrique, en compagnie d'un garde privé et qui, comme tel, pouvait mieux que lui faire l'affaire?... 
Aucune entremise, aucun recours auprès du commissaire adjoint ne fut nécessaire : tous les soldats se défilèrent, refusant la charge d'une telle mission. Aussi, au moment où il se présenta lui-même face à l'intéressé, aux ordres et au garde à vous, tout était déjà réglé. Sans plus attendre, il envoya 'Antar, le commis du buffet, prévenir sa femme de son départ, la priant de lui préparer un casse-croûte dans une serviette et de lui faire porter «le» billet de cinquante piastres, précisant: "Celui glissé dans la taie du traversin ».

Une demi-heure passa....

Tout fut bientôt prêt, la lettre de l'Inspecteur de la Santé rédigée, les formulaires de voyage $^{3}$ remplis. Il ne lui restait plus qu'à poser le pied dans le train et se retrouver dans quelques heures au cœur de l'Egypte.

Chabrâwi avait peine à croire que ce qui venait de se passer était réel, que tout avait été réglé comme ça, aussi facilement et en douceur et qu'il allait bel et bien revoir le Caire, s'y promener, prendre le tram, retrouver les amis, les copains, dîner d'une nîf $a^{4}$ chez Hanafî le rôtisseur. Non, il avait peine à le croire.... Bref, il faisait maintenant route vers la gare, une joie à laquelle il ne croyait pas faisant vaciller ses pas, suivi de plus de cent âmes, chacun le chargeant de veiller sur Zbeïda, d'être patient avec elle.

Le père lui glissa discrètement un rial $^{5}$,le mari dix piastres, et Chabrâwi hocha copieusement la tête, affichant un sourire persistant, leur jurant qu'il répondait d'elle, qu'ils n'avaient aucun souci à se faire et le considèrent comme son frère germain. Le cortège, fendant le village, attirait l'attention de ses habitants et, lorsque voyant Chabrâwi à sa tête, ceux d'entre eux qui le connaissaient lui demandaient où il allait :

- Juste là... répondait-il modestement.

- Là, où ça ? appuyait-on.

Chabrâwi, forçant le ton de l'indifférence :

- Au Caire, quoi !

Souvent lui venait en réponse un «À ta santé, vieux!» Le bonheur lui picotait les entrailles.

6 Après une longue attente, arriva le train du Delta. Il monta en compagnie de Zbeïda qui s'assit calme et tranquille et le train s'ébranla sous la divine protection.

Pour la troisième fois, Chabrâwi palpa les documents qu'il avait rangés avec soin dans sa poche intérieure et, voyant que tout était en ordre de ce côté là et que pour l'heure, c'était du beurre, il dégrafa son large ceinturon, se détendit... Il en avait presque oublié Zbeïda.

8 Après ses haltes, stations et échappées sans fin, le train, long ver noir, entra dans alMansura et, tenant Zbeïda par la main, Chabrâwi traversa la passerelle, scandant inlassablement :

- Sayyida Zeïnab, votre bénédiction!

Il demanda le train du Caire et le trouva à quai, qui l'attendait. Il monta, faisant asseoir Zbeïda près de la fenêtre. Au passage du vendeur de citronnade, il prit deux verres qu'il avala d'un trait et, en offrant un troisième à Zbeïda qui le repoussa d'un geste de dégoût ulcéré, il l'amadoua d'une tape amicale et fit prendre au liquide le chemin déjà par lui emprunté. 
21 Le train s'ébranla avec ses passagers, tous confiants et sereins. Zbeïda regardait par la fenêtre, avec un air d'enfant, un sourire nature à la bouche, tandis que les doigts de Chabrawi claquaient machinalement de bonheur.

Aux abords d'al-Sunbulawên, elle se tourna soudain, se frappa violemment la poitrine et, le regardant d'un air d'étrange suspicion :

- Malheur ! fit-elle.

Rappelé subito des jardins de sa félicité, Chabrâwi lui demanda tout émoi : - Qu'as-tu mon enfant? Qu'y-a-t-il Zbeïda?

Elle ne répondit pas. Elle colla simplement sa main sous son nez et poussa un you-you plein de force et de désinvolture. Une nuée de trilles suivit. Les passagers se retournèrent et un silence de suprême étonnement emplit le wagon. Troublé, légèrement étourdi, Chabrâwi ne dit mot. Après avoir essayé d'avaler sa salive sans la trouver, il donna à Zbeïda une tape à l'épaule, des « excuse-moi ma petite, c'est de ma faute, sois patiente s'il te plaît, pas de scandale », enfin... deux trois mots du genre et Zbeïda se tut.

27 Mais les passagers ne firent point de même. Les commentaires commençaient à fuser en sous-main, la rumeur s'éleva, sans compter les regards, pointés droits sur elle et lui. Il entendit même une femme s'exclamer :

- Ça doit être sa femme, pauvre chérie!

Un rire sonna à l'autre bout du wagon. Tiré de sa somnolence, l'homme assis face à lui toussota et deux gamins grimpèrent debout sur leur siège, s'accoudant au spectacle. Chabrâwi transpirait à ce point que la sueur humectait son uniforme beige. Il tendit la main et attrapa la serviette qu'il avait étendue pour se restaurer puis renouée dans sa position initiale... Inquiété, un voisin demanda :

31 - Cette femme, qu'a-t-elle Monsieur l'agent?

Ayant retrouvé sa langue si ce n'est son assiette, Chabrâwi répondit :

33 - Rien du tout... rien...

34 Puis, après un silence, faisant tournoyer la pointe de ses doigts au voisinage de sa tempe :

$35-$ C'est qu'elle....

36 L'homme acquiesça de tout son corps, comme s'il venait de percer quelque épais mystère. La main de Chabrâwi se mouvait encore que Zbeïda se tourna vers lui et s'exclama de toutes ses forces, les traits vifs et acérés :

37 - Comment ça rien du tout? Comment ça mon petit gars, rien du tout?

38 Avec une réelle anxiété, il la regarda s'approcher, pointant son museau droit sur lui. Il rejeta sa tête en arrière, la collant au revêtement de bois du wagon et interposa la serviette - et son contenu - entre elle et lui. Mais, s'arrêtant brusquement, elle se planta droit sur ses jambes, scruta le plafond de la voiture avec des yeux hagards et lança à tue-tête :

39 - Comment ça, rien ? À bas le maire du village, Ibrahim Abu Shu'lân... A bas le maire et vive Sa Majesté le roi. Vive sa Majesté le roi le Président Mohammed Bey Abu Batta! 
41 Tout le wagon fut mis en alerte et le sommeil quitta brusquement les paupières. L'homme d'en face tira son couffin de dessous le siège et s'éloigna à la hâte. En une seconde, Zbeïda et lui avaient la moitié du wagon pour eux seuls, la totalité des passagers s'étant repliés du côté opposé, appréhendant un malheur. Certains allèrent jusqu'à quitter les lieux, la curiosité retenant le plus grand nombre.

L'uniforme de Chabrâwi était comme lavé de sueur.

Pour forcer Zbeïda à s'asseoir et mettre fin au scandale, il tendit la main, mais elle la lui frappa, puis se ploya en avant, poussant ses trilles et s'exclamant - À bas le maire de chez nous. Vive sa Majesté le roi le Président Abu Batta !... Les rires des vendeurs de "gazeuse " et de cacahuètes retentirent, ralliant ceux des passagers. Chabrâwi ne vit pas de gêne à rire lui aussi. Mais il ne rit pas longtemps. D'un seul coup, l'affaire tourna résolument au sérieux. Avec effarement, il vit Zbeïda étendre le bras et tirer le pan de sa robe, voulant l'ôter, quand c'était son seul vêtement. Il bondit sur elle pour l'arrêter mais elle le repoussa d'une bordée de youyous et ce fut l'empoignade.

S'il finit par avoir le dessus et parvint à la faire asseoir de force, la ligotant avec une écharpe offerte généreusement par l'un des passagers, Zbeïda n'en avait pas moins commis un acte qui le poussa hors de sa retenue. Elle avait jeté son tarbouche par la fenêtre, ce même tarbouche qui n'avait pas bougé de sa tête depuis le premier jour où il était entré en fonction, laissant à nu son crâne blanc planté de quelques cheveux ras.

Zbeïda ne se calma qu'une fois cet acte accompli. Elle ne cessait de pousser ses trilles, ponctuées à chaque fois d' « À bas le maire, vive le Président !... »

À proximité de Belbeis, le calme avait refait chemin vers son esprit et elle se taisait, jusqu'au moment où un petit nombre de voyageurs intrépides entreprirent de rejoindre leurs places. Quant à Chabrâwi, bouillant de colère pour son tarbouche qu'il avait vu s'envoler sous ses yeux, il se faisait un devoir absolu de ne pas la ficher hors du train. Il continua de bouillir jusqu'à l'entrée en gare du Caire.

50 Là, attendant que tous les passagers fussent descendus, il tira violemment Zbeïda et entortilla son bras autour du sien, l'y maintenant serré comme une tenaille. Mais à quoi bon tant de vigueur puisqu'elle allait soumise, moulant ses pas comme de la soie.

51 L'esplanade de la gare l'éblouit, même si les circonstances ne laissaient guère aux souvenirs le loisir d'occuper son esprit. Aussitôt il sauta dans le tram avec Zbeïda, aussi docile et raisonnable qu'on pouvait l'être. Il descendit place d'al-'Ataba, coupa en direction de la rue d'al-Azhar et, avec le rial, acheta un tarbouche, maudissant Zbeïda, son père et son argent véreux. Il se sentait mal à l'aise dans son nouveau couvre-chef, qui pesait sur sa tête aussi lourd qu'un moellon. Il décida, première chose, de faire disparaître Zbeïda de sa vue et de se décharger d'elle, après quoi, le Caire et lui seraient tout entiers l'un à l'autre.

Cette décision le rasséréna et il monta dans un tram où les gens s'entassaient. Puis son esprit s'absorba à repasser les tracas qu'il venait de subir et les heures à venir. Mais, à la moitié du chemin, se reprenant pour jeter un œil sur Zbeïda, il la vit collée à un effendi, la lèvre pendante, avec un air de débilité satisfaite. L'homme, lui, semblait se complaire infiniment à cet état, vaguement occupé à la lecture d'un journal qu'il tenait devant lui. Chabrâwi la rudoya du coude puis, l'écartant, la satisfaction qui baignait son visage le céda à la colère et elle se mit à pousser ses trilles avec des « A bas le maire, 
vive le Président Abu Batta!» Le contrôleur fit stopper le tram en plein trajet et descendre Chabrâwi, l'abreuvant de reproches et de pointes pour être monté avec un tel phénomène.

4 Zbeïda marchait à sa droite et, bien décidée à ne pas interrompre ses you-yous, toute la rue Muhammad Ali s'agglutina bientôt à leur suite et à leurs côtés. Plus les gens affluaient, plus elle criait fort, tandis que Chabrâwi s'abîmait dans une sorte d'absence, le visage rivé vers le sol. Voyant arriver cette troupe en goguette, le soldat de faction à la porte de la préfecture pressentit à l'affaire un tour passionnant. Chabrâwi lui demanda le médecin. Comprenant d'expérience la situation, le soldat compatit, vu qu'il était plus de six heures et qu'il n'y avait plus personne. Aussi Chabrâwi demanda-t-il, en haleine :

- Bien et après ?

À quoi le soldat répondit sereinement :

- Revenez demain!

- Demain ? Comment ça demain?

- Demain matin.

Il fit suivre sa réponse d'une rude semonce qui dispersa les gens, chacun portant dans sa musette un trésor de prodiges... Chabrâwi le supplia, lui demandant s'il n'était pas possible de la laisser là pour la nuit. Comme l'autre le fixait du regard, sans mot dire, Chabrâwi comprit, empoigna Zbeïda et partit...

Dès cet instant, l'acuité du problème commença à lui effleurer l'esprit et il se mit à réfléchir à l'endroit où, nanti d'une telle peste, il allait bien pouvoir passer la nuit. Mais il était fatigué, anéanti, et cela faisait des heures qu'aucune nourriture n'avait pris le chemin de son estomac.

Parvenu à Bab al-Khalq, il franchit la porte du café le plus proche, s'assit et fit asseoir Zbeïda à côté de lui, emboîtant son épaule dans la sienne. Il ne prêta aucune attention aux regards des clients fixés sur elle et lui, pas plus qu'à leurs commentaires...

3 Il demanda un thé et une ta'mîra ${ }^{6}$, absorba le tout et sentit bientôt l'engourdissement courir agréablement dans sa chair. C'est quelque chose, à l'intérieur, qui le faisant soudain s'agiter, s'étirer très fort en arrière puis se plier complètement en avant, le tira de sa griserie. Estimant qu'il ne pourrait pas tenir et qu'il lui fallait se mettre immédiatement en quête de l'endroit où les besoins humains s'accomplissent, il appela le garçon, la face contractée, et l'homme lui désigna un endroit à proximité.

Mais... et Zbeïda ?...

Il jeta un regard alentour et, à la faveur d'une brève conversation nouée sans peine avec son voisin, vêtu d'un paletot et d'une galabiyya, il apprit que celui-ci était inspecteur à la préfecture. Force lui fut de raconter son histoire de $\mathrm{A}$ jusqu'à $\mathrm{Z}$ et de l'achever en priant l'inspecteur de veiller sur Zbeïda, le temps qu'il aille où personne ne pouvait aller pour lui. Aussi, à peine l'homme eût-il frileusement accepté, Chabrâwi s'élança comme un boulet... A son retour, le café s'était transformé en mouled animé par Zbeïda. Chabrâwi l'empoigna brutalement, non sans s'être confondu en excuses auprès de l'inspecteur, et se remit en route sans idée de sa destination. tâchant de lui rappeler le temps d'avant... Mais il était dans un autre univers. Fouillant 
dans sa mémoire, il tomba sur un lointain parent, étudiant en agronomie à l'université et, toujours dans sa mémoire, retrouva le lieu de son domicile. N'ayant jamais vu l'endroit que de jour, il tourna quelques heures dans Guizeh et finit par s'y laisser guider.

Il frappa. Son parent ouvrit, le salua chaleureusement, « où étais-tu, vieux, par Dieu ça fait un bail, comment va la compagnie ? ».... Mais, avant d'avoir pu même aborder le propos, Zbeïda - qui n'avait pas ouvert la bouche pendant tout ce temps - poussa un you-you pétillant.

Chabrâwi la regarda, regrettant de ne pas avoir un couteau sur lui pour l'égorger. Il n'aborda pas le sujet d'un mot. Il se retira simplement en silence, narrant à son parent quelques bribes de son aventure.

Lorsqu'il se retrouva dans la rue, il dit à Zbeïda, lui comprimant le bras avec la ferme intention de le casser :

70 - Et maintenant, qu'est-ce que je vais faire avec toi ? Par Dieu, si tu ne te calmes pas, je t'égorge, quitte à récolter perpète et advienne que pourra!

71 Il continua à jurer, menacer, elle marchant à ses côtés, comme une oie, bille en tête... La perpétuité à laquelle il aspirait lui fit penser au commissariat et il trouva que c'était l'endroit tout désigné pour les héberger elle et lui en cette nuit funeste. L'autobus, et en un saut il se retrouvait devant l'agent de service au poste d'al-Sayyida.

Il re-raconta son histoire qu'il avait appris à ficeler et reficeler....

73 Le shâwîsh hocha paisiblement la tête et dit:

74 - C'est une responsabilité mon cher !.... Vous le savez mieux que personne....

75 A quoi Chabrâwi rétorqua, fumant de colère :

76 - Dans ce cas mettez-nous en garde à vue ! D'une voix lente, le shawish reprit:

77 - C'est que c'est « aussi » une responsabilité...

78 Lorsqu'il quitta le commissariat, il vomissait tout ce qui ressemblait à la responsabilité et à ses amateurs zélés, se flagellant presque et se maudissant d'avoir sauté dans le panneau à pieds joints comme un gros crétin. Reprenant ses esprits, lui vint l'idée de l'hôtel. Mais il la rejeta immédiatement: ils étaient deux, Zbeïda était une femme, dangereuse qui plus est... et ce serait facilement une affaire de cinquante à soixante piastres. Alors, à la grâce de Dieu !...

Chabrâwi n'alla guère plus loin. Il s'assit en tailleur devant la mosquée d'al-Sayyida et tira à lui Zbeïda qui se laissa choir à ses côtés. Seule la pudeur lui interdisait de pleurer. Il ne s'imaginait pas d'autre homme en ce monde traînant pareil poids de misère.

Les illuminés d'al-Sayyida les encerclaient comme des fourmis. Aussi, quand Zbeïda se mit à pousser ses you-yous, sa voix se perdit dans le tintamare des cheikhs et de leur basmala ${ }^{7}$, le caquetage des femmes et la valse des dhikrs. Chabrâwi en fut heureux et ravi. Plus rien dans ce que faisait Zbeïda ne recélait d'étrangeté, d'incongruité. En vérité, c'était lui l'étrange, l'incongru au sein de cette multitude, le seul du même coup à être malheureux. Il aurait voulu perdre le sens pour entrer dans la transe et être heureux, s'égayer comme ces gens... Machinalement, il commença à s'échapper de luimême, de ses peines, de sa colère et à observer ce qui se passait autour de lui. Et ce qui se passait était distrayant. Personne ne demandait à son voisin ce qu'il faisait ou lui interdisait de le faire. 
81 Puis il s'abandonna à la contemplation du cheikh étendu de tout son long à côté de lui, parallèlement au mur et qui, la tête appuyée sur son bras, observait le mouvement avec la plus totale indifférence, le visage baigné d'un tel contentement, d'une telle délectation qu'on l'eût dit le souverain du moment... et de l'instant.

De temps à autre, il baissait la tête, la relevait après une absence et, fixant Chabrâwi du regard, disait d'une voix étirée, profonde et caustique : répondit pas, un autre regarda Zbeïda et s'éclipsa; enfin, une vieille infirmière lui indiqua la réception...

Égypte/Monde arabe, 7 | 1991 

beaucoup en l'interrogeant et en entendant ses réponses délirantes. Chacun de ses rires n'était pas pour Chabrâwi un mince réconfort. Mais le médecin prit finalement l'expression du sérieux et l'informa qu'il n'y avait pas de place pour elle en observation, le notant sur les formulaires. écrit, re-feuilleta les documents et le stupéfia en lui demandant s'il avait amené avec elle un membre de sa famille. C'est un nœud au fond de la gorge qu'il répondit que non. Le médecin l'informa que cette condition était nécessaire pour remplir le certificat $d$ 'hospitalisation et qu'il lui fallait tout simplement retourner d'où il venait. Abasourdi, Chabrâwi répondit blêmissant :

104 - Retourner à la Daqahliyya avec elle ?

105 - Oui.

106 Il rebattit l'idée dans sa tête et trouva que c'était la meilleure solution. Toutefois, s'avisant d'un point important, il dit au docteur :

107 - Impossible mon Bey...je n'ai qu'un seul formulaire de retour... pour moi...

108 - Mon fils, il faut quelqu'un de ses parents.

109 - Je vous en supplie, mon Bey...

110 - Mon fils, c'est une responsabilité que je ne peux pas assumer.

111 Sa rancoeur envers cette responsabilité venait d'exploser. Et, avant que ne l'emporte un vent de révolte qui lui eût fait briser tout ce qui se trouvait devant lui, Zbeïda mit fin au dialogue par un you-you verdoyant, en moins d'un clin d'oeil enleva sa robe de haillons, s'élança brusquement au dehors et se mit à courir dans la cour de la préfecture, laissant l'assistance pieds et mains liés de stupéfaction.

112 Chabrâwi fut le premier à se lancer à sa poursuite avec toute la force dont il disposait. Puis les gens, détenus et soldats l'encerclèrent et Chabrâwi réussit enfin à l'attraper. Lors même qu'elle se débattait, vilipendant le maire, elle le mordit. Chabrâwi poussa un cri, abattit sa main sur son visage et le sang se mit à couler de sa bouche et de ses dents. Après quoi on la ramena dans le bureau du médecin, criant, ruant et trépignant. Puis on apporta la camisole de force et il ne fallut pas moins de quatre personnes pour l'y faire entrer. Zbeïda roula à même le sol, tentant de se dégager, le sang maculant ses dents, son visage et sa bouche au pourtour de laquelle la salive faisait un cercle d'écume.

113 Le médecin rédigea en hâte le certificat sous les yeux d'un Chabrâwi médusé, qui observait Zbeïda, tremblant de tout son corps à la vue de l'état dans lequel elle se mettait. 
114 C'est avec effarement qu'il découvrit, après qu'on l'eût mise dans la camisole, que Zbeïda était folle et qu'elle ne comprenait pas un traitre mot de ce qu'elle disait, qu'elle n'était pour rien dans tout ce qu'elle lui avait fait subir, qu'au surplus elle n'avait rien mangé ni bu pendant tout son séjour avec lui et même depuis le village. Il sentit alors, de la voir ainsi rouler, se tordre, frapper sa tête contre le sol, une étrange pitié s'immiscer en lui.

115 - C'est fait ! lui dit le médecin.

116 Ainsi s'achevait la mission et la « responsabilité » de Chabrâwi. Lui qui s'était imaginé se la faire belle une nuit entière, sitôt achevée sa mission et débarrassé de Zbeïda et de ses malheurs, il reçut la nouvelle comme si elle visait quelqu'un d'autre.

117 Arriva le fourgon. On y fit grimper Zbeïda qui, à la risée générale, avait repris ses youyous et ses «Vive Sa Majesté le Président ». À cet instant, Chabrâwi s'ébranla comme propulsé d'un jet et, priant le chauffeur d'attendre une seconde, courut acheter une baguette de pain et un morceau de halva qu'il tendit au soldat qui l'accompagnait, lui disant d'un ton de supplique :

118 - Tiens, donne lui à manger et veille sur elle.......'il te plait, sur le repos de tes morts, sois bon avec elle...

Et le fourgon s'en alla.

120 Chabrawî s'esquiva et fila droit vers la gare, rassasié du Caire et du monde entier... Par instants, avisant sa paume dont il avait frappé Zbeïda, sa chair se révulsait, en proie à une honte comme jamais il n'en avait ressenti de sa vie.

\section{NOTES}

1. Al-'Obési : rue-quartier d'al-Abbasiyya, parallèle à la rue Ramsès, peu après la gare; alTurgumân (pop. Turkmân) : rue-quartier située derrière l'actuel immeuble du journal al-Ahrâm.

2. Situé autrefois dans le quartier de Sayyeda Zaynab, aujourd'hui démoli.

3. Ceux qui permettent à un fonctionnaire de voyager gratuitement en chemin de fer.

4. Morceau de poitrine de chèvre grillée.

5. Pièce de vingt piastres.

6. Boule de tabac humide posée sur le fourneau de la pipe à eau.

7. De « Bismi-llah », « Au nom de Dieu » (le Bienveillant, le Miséricordieux).

\section{INDEX}

Mots-clés : Idris (Youssef), littérature 